\title{
Evolução do testbed FIBRE
}

\author{
Gustavo Hermínio de Araújo ${ }^{1}$, Marcos Felipe Schwarz', \\ Luiz Eduardo Folly de Campos ${ }^{1}$, Leandro Neumann Ciuffo', \\ Michael Prieto Hernandez ${ }^{1}$, Gustavo Neves Dias ${ }^{1}$.
}

\author{
${ }^{1}$ Rede Nacional de Ensino e Pesquisa (RNP) \\ Rua Lauro Muller, 116 - Sala 1103 - 22.290-906 - Rio de Janeiro - RJ - Brasil \\ \{gustavo.araujo, marcos.schwzarz, luiz.campos, leandro.ciuffo, \\ michael.hernandez, gustavo.dias\}@rnp.br
}

\begin{abstract}
FIBRE (Future Internet Brazilian environment for Experimentation) is a research-driven testbed constructed in the scope of a project funded by the 2010 Brazil-EU Coordinated Call in ICT. The project ended in October 2014. However, in 2015 the Brazilian member institutions, led by the National Research and Education Network (RNP), took over FIBRE's legacy infrastructure to offer the testbed as a service. Currently, funds the secure the FIBRE testbed operational are guaranteed until the end of the year. This paper presents new features and improvements deployed in the last year, as well as strategies to decrease operating costs while new funding opportunities are prospected.
\end{abstract}

Resumo. FIBRE (Future Internet Brazilian environment for Experimentation) é um ambiente para experimentação (testbed) construído no âmbito de um projeto financiado pela Chamada Coordenada Brasil-UE em TIC de 2010. O projeto terminou em outubro de 2014. No entanto, em 2015, as instituições brasileiras membros do projeto, lideradas pela Rede Nacional de Ensino e Pesquisa (RNP), assumiram a infraestrutura legado do testbed FIBRE para oferecer o testbed como um serviço. Atualmente, recursos estão garantidos para a operação do testbed até o final do ano. Este artigo apresenta as novas funcionalidades e melhorias implementadas no ultimo ano, bem como estratégias para reduzir os custos operacionais enquanto novas oportunidades de financiamento são prospectadas.

\section{Introdução}

A avaliação de novas arquiteturas, protocolos, métodos e tecnologias em pesquisas aplicadas em Internet do Futuro dependem de recursos dedicados para experimentação a fim de buscar soluções em áreas como desempenho, medições, segurança, mobilidade, distribuição de conteúdo etc. Diversas iniciativas e projetos financiados por governos e por redes de pesquisa e educação em diferentes países tem sido desenvolvidas desde 2003 [1], implantando infraestruturas dedicadas para dar suporte a pesquisas em Internet do Futuro. O projeto FIBRE (Future Internet testbeds/experimentation between Brazil and Europe) [2], selecionado em 2010 na $1^{\mathrm{a}}$ Chamada Coordenada entre Brasil e Europa em tecnologias de informação e comunicação (TIC), construiu e opera um testbed de larga escala, que atualmente conta com a participação de 15 instituições distribuídas em 
9 estados, mais o Distrito Federal. Atualmente, sendo a maior plataforma para pesquisas aplicadas em Internet do Futuro do Brasil.

\section{Arquitetura do Framework de controle FIBRE}

Os componentes da arquitetura são estruturados em dois níveis: (i) nível locais e (ii) nível global. O nível local é representado pelas ilhas de experimentação. Cada ilha possui controle direto de seus recursos, componentes de gerenciamento e interage com os componentes do nível local através de uma API SFA Aggregate Manager (SFA AM). O nível global é responsável por interagir com o usuário através do Portal de Experimentação, gerenciar as credenciais de usuário através da ClearingHouse e fornecer as interfaces para realizar experimentos através do OMF6. O Portal de experimentação foi desenvolvido especificamente para atender aos requisitos do testbed FIBRE, enquanto, a ClearingHouse foi implementada de acordo com as especificações GENI's. Na Figura 1 é possível observar os níveis da arquitetura do framework de controle do FIBRE e seus principais componentes.

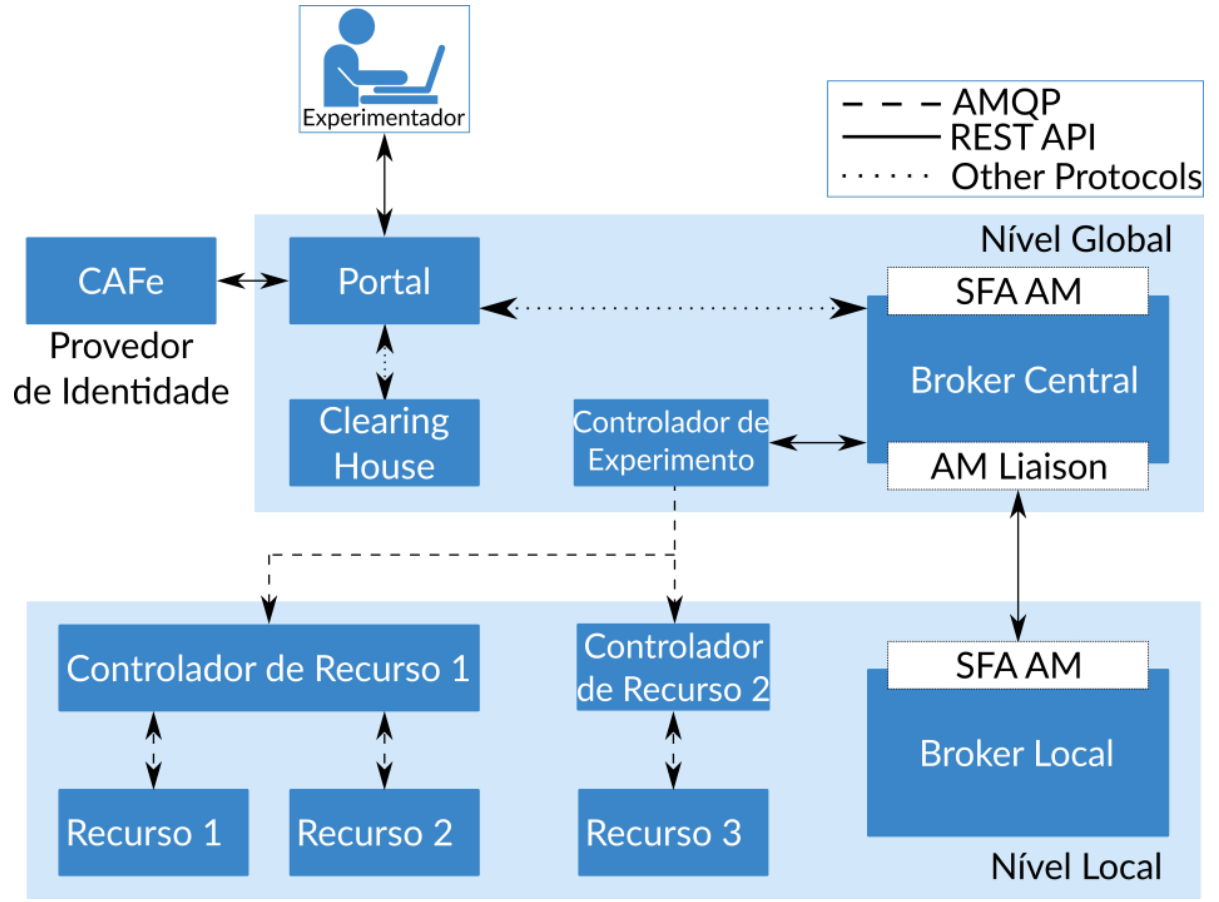

Figura 1 - Desenho da Arquitetura do Framework de controle do FIBRE.

O Central Broker é a entidade responsável por agregar e anunciar recursos disponíveis no testbed, realizar reserva de recursos e por prover os recursos reservados no devido tempo. Para isso, o Central Broker coleta as informações dos recursos nos Brokers Locais através de uma API SFA chama AM Liaison. Os Brokers locais expõem uma API do SFA AM que permite descobrir, agregar e anunciar recursos locais para o intermediário central. Cada ilha deve implantar seu Broker Local para agendar e criar reservas de seus recursos no Portal de Experimentação.

O Controlador de Experimento é a entidade de controle responsável por orquestrar experimentos descritos por scripts escritos em OEDL (OMF Experiment Description Language) [3]. Um sistema de mensagens de publicação e inscrição é 
adotado para lidar com comunicações entre recursos e as entidades que interagem com eles. Os participantes podem criar tópicos, assiná-los e publicar mensagens para eles usando o AMQP 5, um protocolo de camada de aplicação padrão aberto para middlewares orientados a mensagens.

Controladores de recursos intermediam a troca de mensagens entre os recursos locais e da Experiment Controller. Eles são responsáveis por controlar o ciclo de vida dos recursos sob sua governança. Eles criam instâncias de recursos e enviam mensagens de controle arbitrárias para eles. Esses recursos locais podem representar máquinas virtuais, nós sem fio, sensores especializados ou recursos OpenFlow.

\subsection{Portal de Experimentação}

O Portal de Experimentação ${ }^{1}$ é uma interface web criada especificamente para o testbed da FIBRE, que permite aos usuários alocar e interagir com os recursos de cada ilha disponível por meio de um navegador. Experimentadores podem criar e gerenciar projetos compartilhados, construir experimentos e adicione recursos suportados a eles através do portal. O portal de experimentação simplifica o uso da federação porque meio das interações necessárias entre $\mathrm{CH}$, Broker e experimentadores. Na Figura 2 é possível visualizar a interface para a edição de topologias do portal de experimentação.

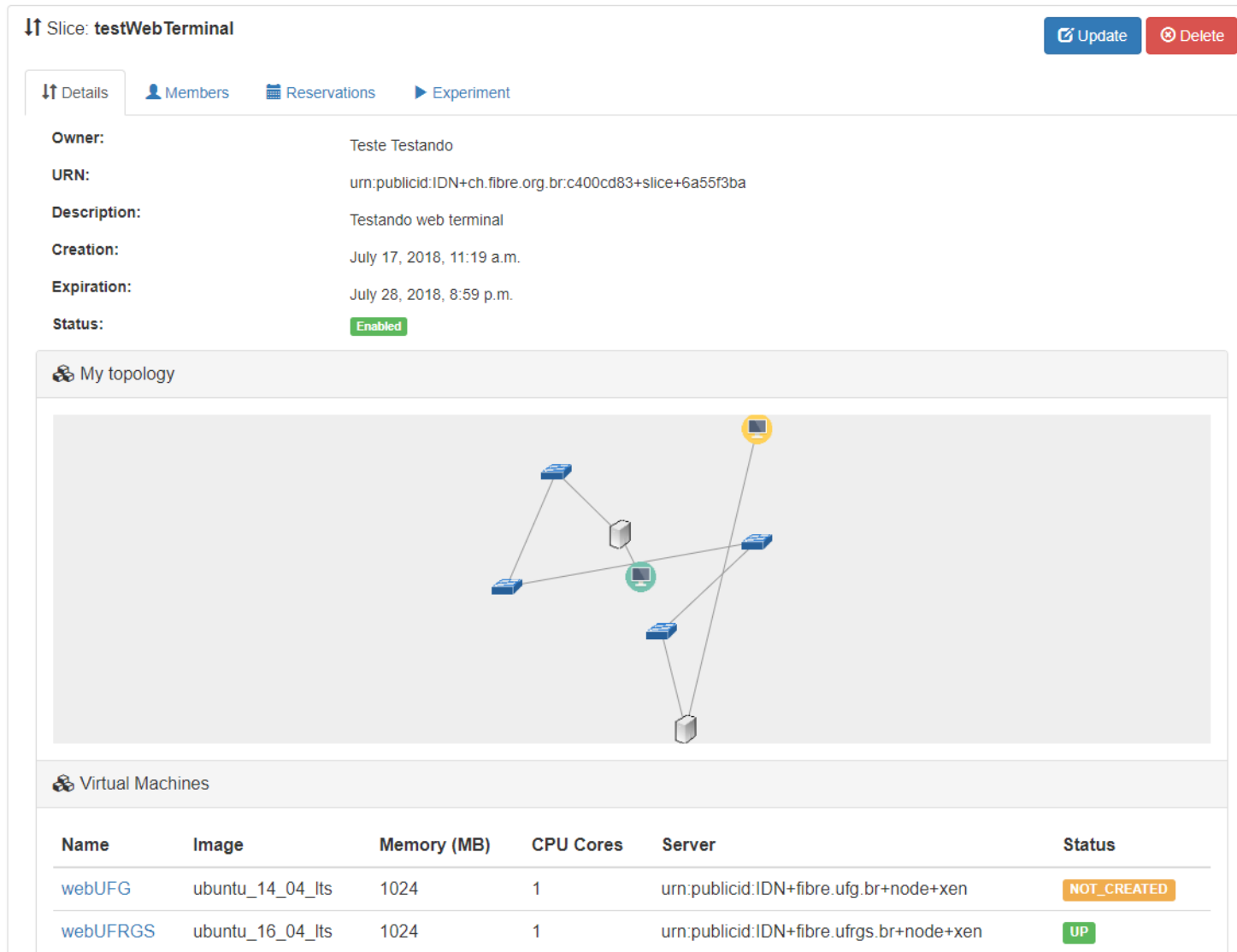

Figura 2 - Tela da Interface para Edição de Topologias.

\footnotetext{
${ }^{1}$ Acessível através da URL: https://portal.fibre.org.br
} 
Para utilizar serviços, os experimentadores devem ser autenticados. Dada a diversidade de usuários que pretendemos alcançar com a FIBRE, os experimentadores podem usar a autenticação concedida pela federação brasileira de identidade acadêmica chamada Comunidade Acadêmica Federada (CAFe). No entanto, o portal também suporta autenticação local para pesquisadores que não estão contemplados com uma conta federada. O Portal de Experimentação interage com o OMF6 Central Broker para a descoberta e a reserva de recursos por meio de uma API REST exposta pelo Central Broker.

\section{Evolução do Backbone FIBREnet}

Os ambientes de pesquisa do testbed FIBRE, conhecidos como ilhas de experimentação, são interconectados por uma infraestrutura SDN denominada FIBREnet, composta de switches SDN OpenFlow e enlaces de rede em camada 2 sobrepostos ao backbone da RNP (Rede Ipê). A FIBREnet representa a maneira ideal de conectar as ilhas, através de links dedicados. Ela permite a execução de experimentos de forma federada, utilizando recursos de duas ou mais ilhas no mesmo experimento, e oferece a programabilidade de seus switches OpenFlow, interconectados em malha parcial, aos próprios experimentadores, permitindo que possam personalizar a arquitetura de rede que pretendem utilizar entre as ilhas participantes do experimento.

Ao longo da evolução do projeto, de 2011 a 2018, a FIBREnet implantou 10 switches, instalados em Pontos de Presença da RNP de diferentes estados, e 15 enlaces de $1 \mathrm{Gbps}$ entre eles, seguindo a arquitetura original do projeto FIBRE para a FIBREnet. Devido a algumas limitações, como a falta de suporte a novas versões do protocolo OpenFlow (1.3), o limite da taxa máxima de vazão em $1 \mathrm{Gbps}$, e à solução específica de virtualização utilizada originalmente, foi elaborado um novo conjunto de soluções para que a FIBREnet pudesse atender aos requisitos de um testbed de experimentação com suporte às novas tecnologias e protocolos recentes e futuros. A nova solução, chamada de SDN Overlay, compreendeu o uso de equipamentos com arquitetura de hardware genérica e aberta, conhecidos como whiteboxes, o uso de software de código aberto, como o sistema operacional Linux, o switch virtual Open vSwitch [5] e o driver de aceleração de processamento de pacotes DPDK [4], e o desenvolvimento de um gerenciador de redes virtuais para administração e operação das redes virtuais que seriam ativadas sobre essa infraestrutura, chamado SOLO. Assim, a nova arquitetura da FIBREnet seria provida por switches SDN Open vSwitch de versão recente, com suporte a OpenFlow nas versões de 1.0 a 1.5, e herdando todas as demais funcionalidades providas pela pilha de software do Open vSwitch.

A arquitetura da SDN Overlay foi viabilizada como parte do projeto IDS (Infraestrutura Definida por Software), e o testbed FIBRE se tornou então seu primeiro e principal utilizador, após a migração da arquitetura original FIBREnet para esse novo modelo. Inicialmente, os whiteboxes foram implantados nos mesmos PoPs onde já estavam hospedados os switches FIBREnet. Em seguida, eles foram adicionados à rede do FIBRE com portas físicas dedicadas, porém servindo apenas como trânsito de tráfego entre o backbone da RNP e os switches originais. Então foram desenvolvidos os switches virtuais Open vSwitch nos whiteboxes, replicando de forma fiel as características dos switches físicos da rede original, como DPIDs (identificador de cada switch OpenFlow), condições de interfaces e seus respectivos circuitos, com o intuito de 
reconstruir uma topologia idêntica. Como último passo, a manipulação SDN foi deslocada dos switches originais para os novos switches, retirando a parte OpenFlow dos switches originais nos PoPs e conectando suas réplicas virtuais nos whiteboxes às interfaces físicas destinadas ao FIBRE. A migração foi feita de gradualmente, de forma que ao início de 2019 todos os switches SDN FIBREnet já eram providos pela nova arquitetura Open vSwitch. Muitos dos switches originais ainda continuam na topologia do testbed, servindo como trânsito para o tráfego que usa links ópticos para chegar até as ilhas. Por fim, os experimentadores contam agora com a mesmas funcionalidades SDN providas pela arquitetura anterior, porém com a vantagem de uma nova infraestrutura que poderá ser utilizada para a evolução do testbed como um todo, incluindo novas versões de protocolos como o OpenFlow 1.3.

A Figura 3 ilustra a nova arquitetura da FIBREnet. A camada de abaixo representa os 10 equipamentos Whitebox físicos, implantados nos mesmos PoPs da FIBREnet. A segunda camada, com enlaces em vermelho, se refere à rede virtualizada replicando a FIBREnet, criada para garantir o funcionamento transparente dos serviços oferecidos pelo FIBRE atualmente e permitir a migração gradual para esse novo modelo. A terceira camada, com 3 enlaces em verde, representa uma outra rede virtual de experimentação, instanciada por meio do novo sistema de controle e gerência do FIBRE (OMF 6).

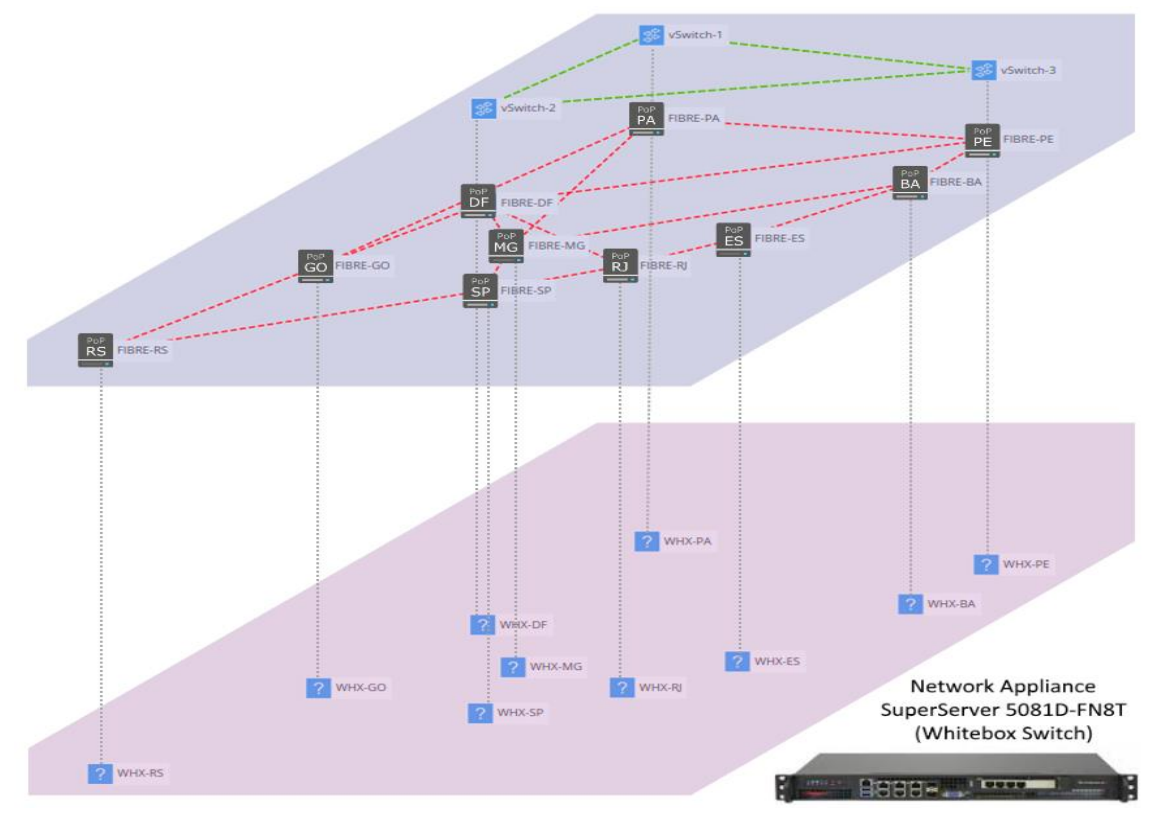

Figura 3 - Arquitetura da FIBREnet e os switches whitebox físicos utilizados na implantação.

\section{Trabalhos Futuros}

O Testbed FIBRE permaneceu em contínua evolução ao longo do último ano, tendo como iniciativas em andamento as apresentadas a seguir. 


\subsection{Automação Integrada da Operação e Desenvolvimento}

Considerando a atual infraestrutura do FIBRE, que consiste em mais de 100 equipamentos em diferentes categorias de propósitos (switches OpenFlow, switches ToR, servidores de virtualização, servidores NetFPGA, servidores perfSONAR e múltiplas máquinas virtuais) distribuídos em 17 ilhas, muito do trabalho de operação está direcionado para a implantação de novas ilhas, documentação e capacitação de novos operadores, manutenções e atualizações de infraestrutura.

Dado este contexto, a equipe de operação do FIBRE tem focado em adotar boas práticas de automação para melhorar a agilidade e a replicabilidade destas atividades. Após o estudo de diversas ferramentas, foram definidos quatro estágios de automação: I) máquinas físicas (baremetal); II) configuração; III) conteinerização e IV) integração e entrega contínua $(\mathrm{CI} / \mathrm{CD})$.

O primeiro estágio consiste em habilitar a operação remota e automatizada de todos os ativos físicos, incluindo acesso ao terminal das máquinas, controle do fornecimento de energia e reinicializações, e a automação do provisionamento do sistema operacional da máquina. Para alcançar tal objetivo, foi escolhida a solução aberta MAAS da Canonical dado sua facilidade de uso e conjunto de funcionalidades. $O$ MAAS foi validado para controlar todos os servidores e switches whitebox do FIBRE, ele será implantado em cada PoP da RNP e irá remotamente instanciar novas ilhas ou atualizar as ilhas existentes.

O segundo estágio é a gerência de configuração e consiste em preparar o Sistema Operacional para atender a todo o conjunto de ferramentas e requisitos definidos pelo FIBRE. Nesta etapa, a ferramenta selecionada foi o Ansible, dado a sua rápida curva de aprendizado e por suportar ambos equipamentos de rede e servidores. Após o servidor ser instalado na etapa anterior, o Ansible executa um conjunto pré-definido de receitas (playbooks) que são responsáveis pela instalação das aplicações e configurações de cada equipamento como instalação do docker, configuração de rede e tunings, deixando o ambiente pronto para ser usado. As receitas são criadas de forma a separar os parâmetros mínimos de cada ambiente como IPs, sistema operacional e credenciais e construir um conjunto de passos bem definidos que serão aplicados e reaproveitados em toda a infraestrutura.

O terceiro estágio consiste em substituir as máquinas virtuais de serviço do FIBRE por containers, de forma a reduzir o consumo de recursos, reduzir a quantidade de elementos gerenciáveis e facilitar a utilização de conceitos como infraestrutura imutável, que simplifica a previsibilidade e agilidade dos processos de mudança. Inicialmente será utilizado Docker como solução para executar containers, mas já está em estudo a adoção do Kubernetes, que apesar da sua difícil curva de aprendizado, possibilita uma orquestração de containers mais completa e novas funcionalidades como escalabilidade horizontal e auto regeneração (self-healing). Serão utilizados containers pré criados e testados por terceiros ou pelo próprio FIBRE usando o quarto estágio.

O quarto estágio é a integração e entrega contínua $(\mathrm{CI} / \mathrm{CD})$, que consiste em integrar o código desenvolvido ao projeto principal na mesma frequência com que as funcionalidades são desenvolvidas, sendo feito muitas vezes ao dia ao invés de apenas uma vez. O objetivo principal de utilizar a integração contínua é verificar se as 
alterações ou novas funcionalidades não criaram novos defeitos no framework de controle FIBRE. Para utilizar o processo de integração contínua no contexto do testbed FIBRE, utilizamos as seguintes ferramentas:

- GitLab: realiza o controle de versão do framework de controle do testbed FIBRE.

- Jenkins: realiza a orquestração e automatização do processo de desenvolvimento de software e integração contínua.

- Nexus: utilizado como gerenciador de repositórios onde são armazenados os artefatos (e.g., instaladores, scripts de configuração) do framework de controle.

- SonnarQube: verifica a qualidade do código que foi enviado ao GitLab.

- Ansible: realiza o provisionamento de software, gerenciamento de configuração e implementação de software.

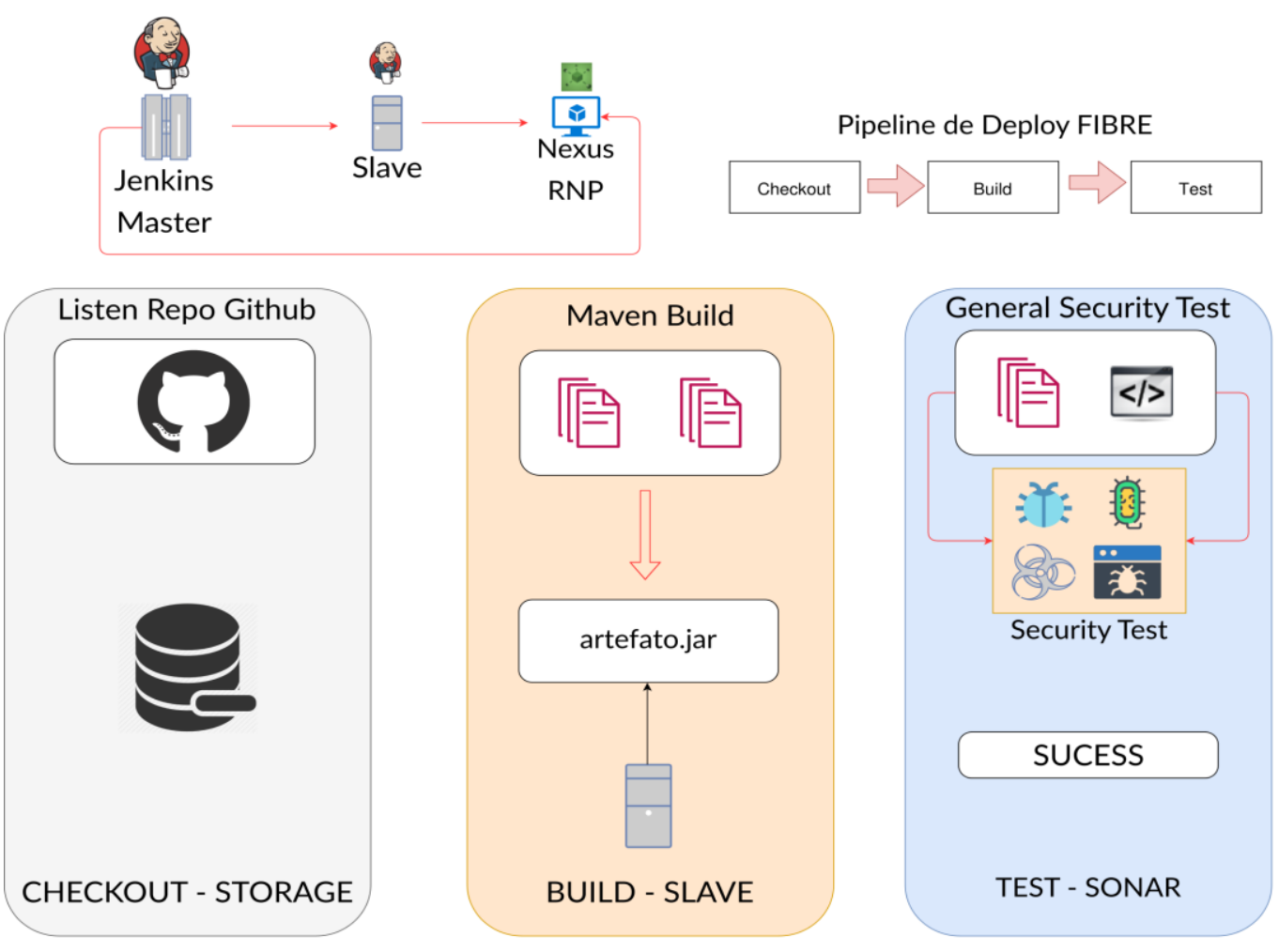

Figura 4 - Fluxo proposto para integração e entrega contínua

O fluxo completo e as ferramentas de integração contínua são exemplificadas na Figura 4. Primeiramente o desenvolvedor realiza um commit do novo código na ferramenta GitLab. Neste estágio são identificadas as modificações realizadas no código e é feito o seu armazenamento. Em seguida é iniciado o estágio de build onde o código é compilado e/ou construído e então um artefato é gerado e armazenado no gerenciador de repositório NEXUS. Em seguida, o código é submetido ao estágio de teste, onde o SonarQube realiza testes genéticos para verificar a integridade do código. Esses testes vão desde ataques de SQL Injection até DDoS e Brute Force. Ou seja, são testes 
genéricos, que podem ser executados em qualquer aplicação, uma vez que têm por objetivo encontrar problemas mais comuns e básicos relacionados à segurança da informação. Por fim, os artefatos são copiados e instalados em seus respectivos alvos (e.g., as ilhas de experimentação) através da ferramenta Ansible.

\subsection{FIBRE in a box/FIBRE in a laptop}

Os esforços de automação da infraestrutura, além das vantagens apresentadas na seção anterior, possibilitam oferecer uma versão do FIBRE (i.e., FIBRE-In-a-Box) com baixo consumo de recursos, orientada a experimentação em um contexto local (e.g., laptop), abstraindo ao pesquisador aspectos como autenticação federada e o uso de um ambiente compartilhado.

Do ponto de vista da arquitetura o FIBRE-In-a-Box adiciona aos componentes do plano de controle do FIBRE, uma camada de rede emulada e nós de computação, encapsulados em containers. Desta forma a solução como um todo permanece autocontida e isolada sem possuir dependências de bibliotecas ou configurações externas.

FIBRE-In-a-Box permitirá aos experimentadores o planejamento, desenvolvimento e a execução de testes das aplicações de forma ágil, em ambiente local, sem as restrições do tempo alocado para o experimento ou os recursos disponíveis no ambiente de experimentação federado.

Uma vez que o experimentador validar o correto funcionamento da aplicação e precisar de um ambiente mais robusto com comportamento determinístico, no qual o experimento possa ser reprodutível e com resultados de valor científico, FIBRE-In-aBox possibilitará a implantação do experimento no FIBRE sem intervenção do usuário.

Com a disponibilização do FIBRE-In-a-Box para o uso da comunidade científica pretende-se aproximar os pesquisadores do paradigma das redes definidas por software através de plataformas de baixo consumo de recursos, flexíveis e de fácil instalação que permitem o desenvolvimento de PoCs ao mesmo tempo que se integram com ambientes de experimentação de alta confiabilidade.

\subsection{Expansão e experimentação de tecnologias associadas à Industria 4.0}

Duas novas ilhas de experimentação deverão ser integradas em até 1 ano e estarão hospedadas no Laboratório de Engenharia de Sistemas (LABESys), localizado na UFSC e no Laboratório de Robótica (LAR), localizado na UFBA. Essas duas ilhas disponibilizarão um conjunto de sensores e atuadores que poderão ser programados e parametrizados pelos usuários do testbed.

Essa expansão será viabilizada por meio do projeto IND4FIBRE, coordenado pelo INESC P\&D Brasil e financiado pela Agência Brasileira de Desenvolvimento Industrial (ABDI). O projeto IND4FIBRE objetiva fomentar a adoção de tecnologia associada à Industria 4.0 através da instalação de dois ambientes para experimentação, no INESC P\&D Brasil e seus conveniados da Rede INESC BRASIL, as universidades 
UGF e UFBA, composta de sensores e atuadores que poderão ser programados e parametrizados pelos usuários do testbed, implantação de um repositório de dados para publicação e armazenamento dos dados coletados pelos sensores, adaptação do software FlexSim para consumir os dados publicados no repositório. A Figura 5 ilustra os componentes envolvidos na solução.

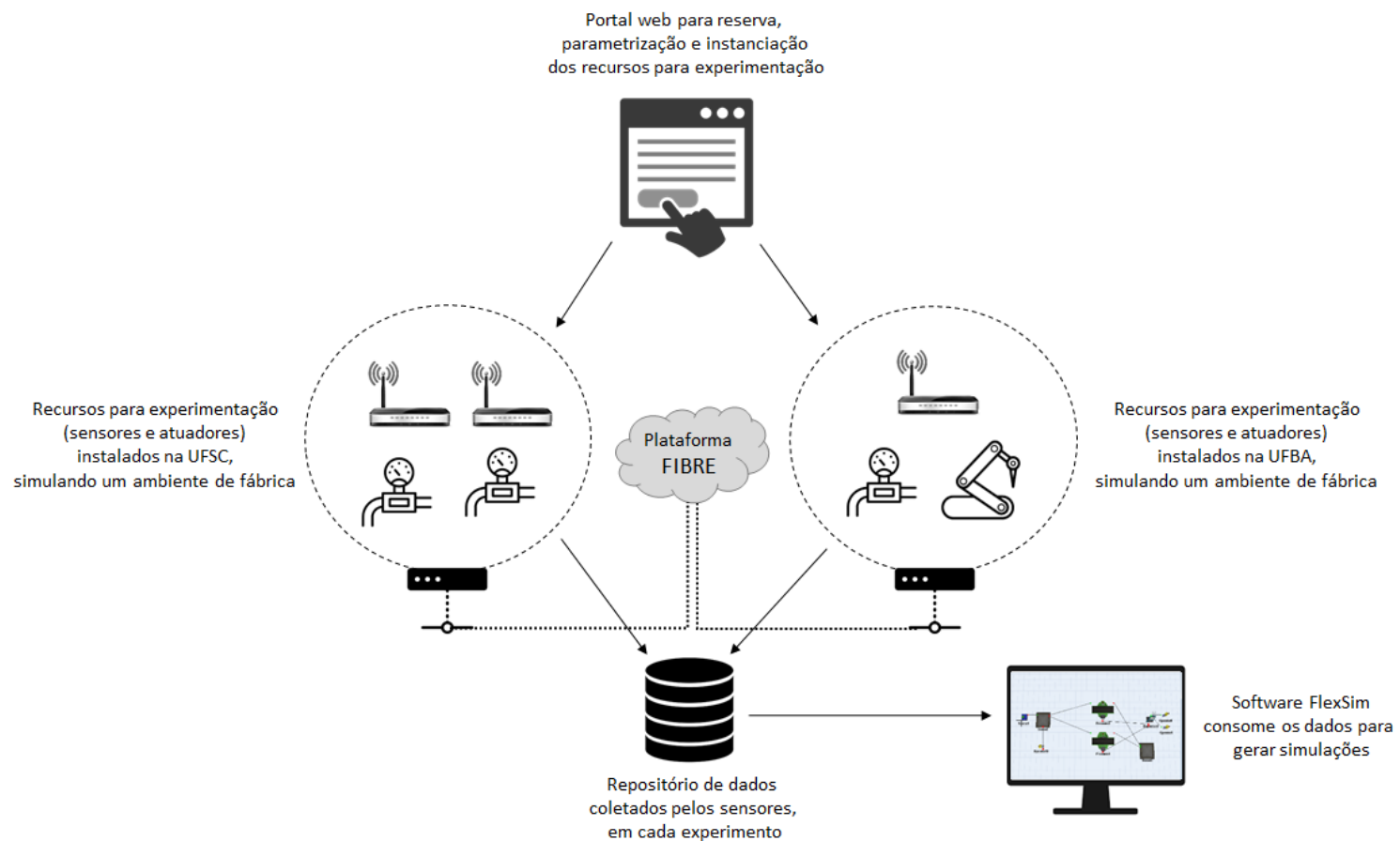

Figura 5 - Diagrama com os componentes da plataforma de experimentação.

\section{Conclusão}

Este artigo apresentou as evoluções implementadas no testbed FIBRE no último ano, desde a apresentação realizada no WPEIF $2018^{2}$. De forma a contribuir para a sustentabilidade do FIBRE, algumas ações estão sendo empreendidas, conforme relatadas no artigo: (i) Automação integrada da operação e desenvolvimento; (ii) migração da arquitetura original da rede FIBREnet para nova solução SDN Overlay; (iii) redução da barreira de entrada de novos experimentadores através da abordagem "FIBRE in a box" e (iv) expansão dos recursos programáveis disponíveis para experimentação através do apoio à submissão de projetos de pesquisa que façam uso da infraestrutura do FIBRE, como no caso do projeto IND4FIBRE.

Outra ação em andamento é a integração da base de usuários do FIBRE ao do novo testbed CloudNEXT ${ }^{3}$, que permitirá experimentação de novas arquiteturas, plataformas e aplicações em computação em nuvem, com acesso direto ao hardware da

\footnotetext{
${ }^{2}$ https://www.fibre.org.br/o-fibre-no-sbrc-2018/

${ }^{3}$ http://cloudlab-brasil.rnp.br/testbed
} 
infraestrutura. A proposta é que usuários do FIBRE não necessitem criar nova conta para acessar a infraestrutura do ClodNEXT. Esse novo testbed é fruto de um projeto financiado pela FINEP que está próximo de sua conclusão.

A integração do FIBRE com outros ambientes para experimentação, tais como as infraestruturas legado dos projetos NECOS, 5GINFIRE e FUTEBOL, já começaram a ser mapeadas e foram materializadas na forma de um projeto de pesquisa submetido ao edital "Chamada de Propostas FAPESP / MCTIC - 2018", ainda aguardando resultado.

Por fim, novas ações de promoção e incentivo ao uso do testbed FIBRE precisarão ser empreendidas e focadas não apenas para a comunidade acadêmica, mas também para micro e pequenas empresas do setor de TICs.

\section{Referências}

[1] PAN, J., PAUL, S. e JAIN, R. A survey of the research on future internet architectures. IEEE Communications Magazine, vol. 49, no. 7, págs. 26 a 36, julho de 2011. DOI: 10.1109/MCOM.2011.5936152. Acesso em: 25/03/2018. Disponível em: <http://ieeexplore.ieee.org/document/5936152/>

[2] SALMITO, T., CIUFFO, L., MACHADO I., SALVADOR S., STANTON, M. et al. FIBRE - An International Testbed for Future Internet Experimentation. Simpósio Brasileiro de Redes de Computadores e Sistemas Distribuídos - SBRC 2014, maio 2014, Florianópolis, Brazil. p. 969, 2014. Acesso em: 25/08/2018. Disponível em: $<$ https://hal.archives-ouvertes.fr/hal-00990392>

[3] T. Rakotoarivelo, M. Ott, G. Jourjon, and I. Seskar. Omf: A control and management framework for networking testbeds. SIGOPS Oper. Syst. Rev., 43(4):54-59, Jan. 2010.

[4] INTEL, D. P. D. K. Data Plane Development Kit. 2014.

[5] PFAFF, Ben et al. Extending Networking into the Virtualization Layer. In: Hotnets. 2009. 